14,15

\title{
Особенности релаксации тензора напряжения в микроскопическом объеме нематической фазы под действием сильного электрического поля
}

\author{
(C) A.B. Захаров
}

Институт проблем машиноведения РАН, Санкт-Петербург, Россия

E-mail: alexandre.zakharov@yahoo.ca

(Поступила в Редакцию 30 мая 2017 г.)

\begin{abstract}
Предложено численное исследование новых режимов переориентации поля директора $\hat{\mathbf{n}}$, скорости $\mathbf{v}$ и компонент тензора напряжения $\sigma_{i j}(i j=x, y, z)$ нематического жидкого кристалла (ЖК), инкапсулированного в прямоугольный канал, под действием сильного электрического поля $\mathbf{E}$, направленного под углом $\alpha\left(\sim \frac{\pi}{2}\right)$ к горизонтальным ограничивающим поверхностям ЖК-канала. Численные расчеты, выполненные в рамках нелинейного обобщения классической теории Эриксена-Лесли, показали, что при определенных соотношениях моментов и импульсов, действующих на единицу объема ЖК-фазы, и в случае $E \gg E_{\text {th }}$, в процессе переориентации $\hat{\mathbf{n}}$ могут возникнуть переходные периодические структуры, если соответствующая мода искажения обладает наибыстрейшим откликом и, таким образом, подавляет все остальные моды. Возникающие при этом вращающиеся домены способствуют уменьшению скорости диссипации энергии и тем самым создают более выгодные, по сравнению с однородным поворотом, режимы переориентации поля директора.
\end{abstract}

Работа выполнена при финансовой поддержке РФФИ (грант № 16-02-00041a).

DOI: 10.21883/FTT.2018.02.45400.177

\section{1. Введение}

В последнее время все чаще методы микро- и нанофлуидистики находят применение при исследовании процессов транспортировки и сортировки нанолитровых объемов жидкокристаллических (ЖК) материалов в разветвленных каналах и капиллярах (lab-on-chip-system) под действием внешнего электрического поля (электрокинетика) [1,2]. С другой стороны, ЖК-материалы используются при создании новых семейств сенсоров, термоиндикаторов и детекторов давления $[3,4]$. На формирование течений в этих микро- и наноразмерных каналах и капиллярах оказывают сильное влияние внешние силы, такие как электрические поля и механические воздействия, а также геометрия ЖК-каналов и характер приповерхностной ориентации поля директора. В связи с этим всестороннее исследование динамических режимов переориентации поля директора и релаксации компонент тензора напряжения в микроскопических объемах ЖК-фаз под действием сильного электрического поля $(\geq 1 \mathrm{~V} / \mu \mathrm{m})$ позволит понять и, как следствие, улучшить динамические характеристики сенсоров, терморегуляторов и датчиков, применяемых в медицинской диагностике и биологических лабораториях на чипах.

Одним из менее затратных, и в тоже время кратчайших, путей к пониманию особенностей динамической переориентации поля директора $\hat{\mathbf{n}}(\mathbf{r}, t)$ в ЖК-фазе, инкапсулированной в микро- и наноразмерные объемы, под действием сильного электрического поля $\mathbf{E}$, является теоретическое моделирование гидродинамических и релаксационных процессов, возникающих в этих молеку- лярных системах. В случае отсутствия электрического поля $(E=0)$ ориентация ЖК-фазы, помещенной в канал, определяется граничной ориентацией (гомеотропной, планарной и наклонной) молекул ЖК-материала. В момент включения электрического поля $\mathbf{E}$, направленного, например, практически перпендикулярно планарно ориентированному нематику, молекулы, образующие ЖК-фазу, начинают переориентацию вдоль Е (рис. 1). Это приводит к возникновению конкуренции с приповерхностными силами, которые транслируются в объем

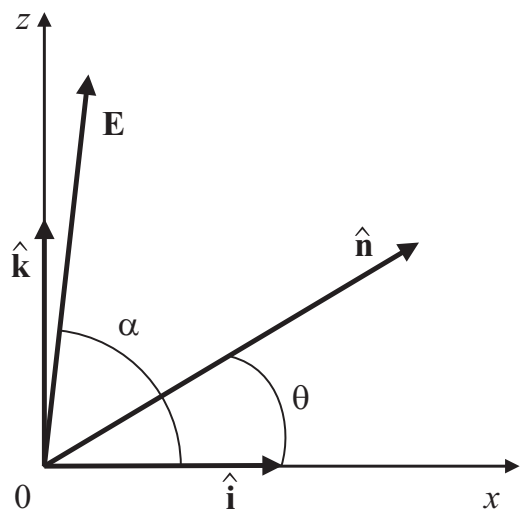

Рис. 1. Координатная система. Орт $\hat{\mathbf{i}}$ направлен параллельно, a орт $\hat{\mathbf{k}}$ перпендикулярно к нижней ограничивающей поверхности ЖК-канала. Вектор электрического поля Е и вектор поля директора $\hat{\mathbf{n}}$ направлены под углом $\alpha$ и $\theta$ к нижней горизонтальной ограничивающей поверхности ЖК-канала соответственно. 


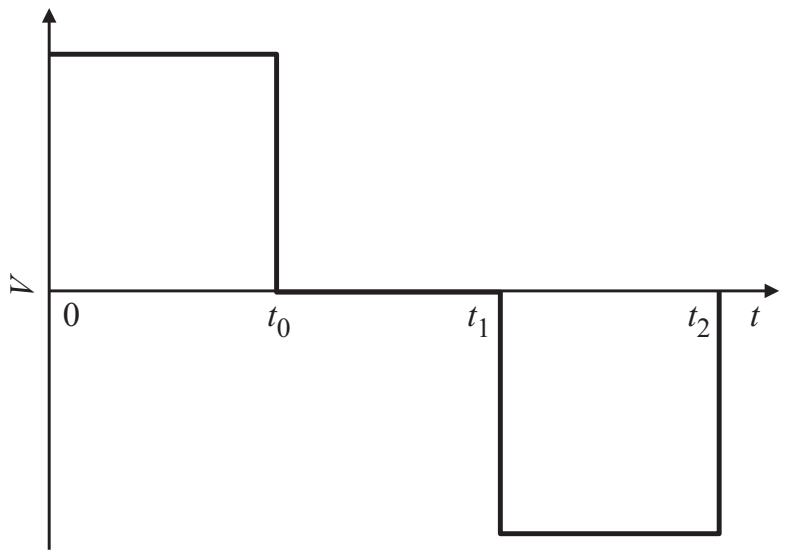

Рис. 2. Три режима включения электрического поля $\mathbf{E}$.

ЖК-фазы посредством ориентационной упругости, присущей всем ЖК-материалам. При значениях величины поля $E>E_{\text {th }}$ молекулы ЖК-фазы однородно разворачиваются в сторону вектора $\mathbf{E}[5]$. Здесь $E_{\text {th }}$ некоторое пороговое значение внешнего электрического поля, при достижении которого начинается переориентация поля директора (переход Фредерикса [5]). Эта величина зависит от конкретного ЖК-материала и его размеров. В случае $E \gg E_{\text {th }}$ ЖК-система может быть выведена из равновесного состояния и любые малые отклонения начальной ориентации поля директора, вызванные, например, термофлуктуациями, могут начать экспоненциально расти со своими коэффициентами роста, которые обратно пропорциональны некоторой эффективной вращательной вязкости ЖК-материала [6]. При классическом переходе Фредерикса однородный поворот молекул ЖК-фазы происходит в плоскости, образованной полем директора $\hat{\mathbf{n}}$ и полем $\mathbf{E}$, и характеризуется сравнительно большим эффективным коэффициентом вращательной вязкости и отсутствием течения ЖК-фазы. С другой стороны, в случае $E \gg E_{\text {th }}$ в процессе переориентации поля директора $\hat{\mathbf{n}}$ могут возникнуть переходные квазипериодические структуры, если соответствующая мода искажения обладает наибыстрейшим откликом и, таким образом, подавляет все остальные моды, в том числе и однородные [7]. При квазипериодическом искажении ЖК-фазы появляется сдвиговая вязкость, уменьшающая общую эффективную вращательную вязкость, связанную с переориентацией поля директора. Возникающие при этом вращающиеся домены способствуют уменьшению эффективной вязкости, характеризующей скорость диссипации энергии, и тем самым создают более выгодные, по сравнению с однородным поворотом, режимы переориентации поля директора.

Исследование этих новых состояний будет проведено в рамках нелинейного обобщения классической теории Эриксена-Лесли $[8,9]$ с учетом балансов массы, импульсов и моментов, действующих на единицу объема ЖК-материала. Численные исследования характера переориентации поля директора и формирование ре- лаксационных режимов компонент тензора напряжения в микроразмерных ЖК-каналах, образованных молекулами 4-n-пентил-4'-цианобифенила (5ЦБ), будут проведены для трех динамических режимов. Первым будет изучен режим (режим I) включения электрического поля $\mathbf{E}>0$, направленного вдоль оси $z$, в течение времени $0<t<t_{0}$ (см. рис. 2), т. е. практически перпендикулярно планарно ориентированному нематику. Вторым будет изучен режим (режим II), когда электрическое поле $(E=0)$ выключено в течение времени $t_{0} \leq t<t_{1}$. И наконец, третим будет изучен режим (режим III) включения электрического поля $\mathbf{E}<0$ в отрицательном направлении вдоль оси $z$, в течение времени $t_{1} \leq t<t_{2}$ (см. рис. 2).

\section{2. Основные уравнения}

Рассмотрим длинный прямоугольный ЖК-канал с размерами $2 L$ и $2 d(L \gg d)$, ограниченный твердыми горизонтальными и вертикальными поверхностями. Допустим, что директор планарно ориентирован на горизонтальных ограничивающих поверхностях и гомеотропно на вертикальных, причем рассмотрим случай, характеризующийся сильным сцеплением ЖК-молекул со всеми твердыми поверхностями. В этом случае система координат отсчитывается от центра ЖК-канала так, что ось $X$ и орт $\hat{\mathbf{i}}$ совпадают с направлением директора на нижней горизонтальной поверхности $\left(\hat{\mathbf{i}} \| \hat{\mathbf{n}}_{z=-d}\right)$, в то время как ось $Z$ и орт $\hat{\mathbf{k}}$ направлены ортогонально $\left(\hat{\mathbf{k}} \perp \hat{\mathbf{n}}_{z=-d}\right)$, а орт $\hat{\mathbf{j}}=\hat{\mathbf{k}} \times \hat{\mathbf{i}}$ (см. рис. 1). Таким образом, в начальный момент времени мы имеем дело с планарно и однородно ориентированным ЖК-образцом, образованным молекулами 5ЦБ, притом, что вектор электрического поля $\mathbf{E}=E_{x} \hat{\mathbf{i}}+E_{z} \hat{\mathbf{k}}=E(z) \cos \alpha \hat{\mathbf{i}}+E(z) \sin \alpha \hat{\mathbf{k}}$ направлен под углом $\alpha\left(\sim \frac{\pi}{2}\right)$ к горизонтальным поверхностям ЖК-канала. После включения электрического поля $\mathbf{E}$, направленного практически ортогонально к планарно и однородно ориентированному ЖК-каналу, в ЖК-фазе начинается переориентация полярных молекул и, как следствие, переориентация $\hat{\mathbf{n}}(\mathbf{r}, t)$ вдоль направления вектора E. Этот процесс переориентации сопровождается формированием поля скорости $\mathbf{v}(\mathbf{r}, t)$ в объеме ЖК-фазы.

Будем предполагать, что переориентация поля директора $\hat{\mathbf{n}}=n_{x} \hat{\mathbf{i}}+n_{z} \hat{\mathbf{k}}=\cos \theta(x, z, t) \hat{\mathbf{i}}+\sin \theta(x, z, t) \hat{\mathbf{k}}$ под действием электрического поля $\mathbf{E}$ осуществляется в плоскости XOZ. Здесь $\theta-$ угол, образованный директором $\hat{\mathbf{n}}$ и ортом $\hat{\mathbf{i}}$ (см. рис. 1). Таким образом, формирование гидродинамического течения $\mathbf{v}(\mathbf{r}, t)$, обусловленного переориентацией поля директора $\hat{\mathbf{n}}$, под действием сильного электрического поля $\mathbf{E}$ может быть описано в рамках нелинейного обобщения классической теории Эриксена-Лесли $[8,9]$, которое учитывает баланс массы, импульсов и угловых моментов, действующих на единицу ЖК-фазы, а также закон сохранения зарядов. Принимая во внимание микроскопические размеры ЖК-канала, мы можем предположить, что плотность 
ЖК-системы постоянна, и мы имеем дело с несжимаемой жидкостью. Условие несжимаемости ЖК-материала $\nabla \cdot \mathbf{v}=0$, или $u_{x}+w_{z}=0$, выполняется за счет введения безразмерной функции тока $\bar{\psi}=\frac{4 \gamma_{1}}{\epsilon_{0} \epsilon_{a}} \frac{1}{U^{2}} \psi$, где безразмерные компоненты вектора скорости $\mathbf{v}=u \hat{\mathbf{i}}+w \hat{\mathbf{k}}$ выражены через $\psi$ как $u=\frac{\partial \psi}{\partial z}=\psi_{, z}$ и $w=-\frac{\partial \psi}{\partial x}=-\psi_{, x}$ соответственно. Здесь и далее мы используем безразмерные пространственные переменные $\bar{x}=x / d$ и $\bar{z}=z / d$, а также безразмерное время $\tau=\frac{\epsilon_{0} \epsilon_{a}}{\gamma_{1}^{2}}\left(\frac{U}{2 d}\right)^{2}$, причем в дальнейшем верхняя черта над пространственными переменными и функцией тока будет опущена, $\epsilon_{0}$ диэлектрическая проницаемость вакуума, $\epsilon_{a}$ - диэлектрическая анизотропия ЖК-системы, $\gamma_{1}$ и $\gamma_{2}-$ коэффициенты вращательной вязкости, а $U=2 E d-$ величина напряжения. Баланс моментов, действующих на единицу объема ЖК-фазы, в безразмерном виде может быть записан как $[7,10]$

$$
\begin{aligned}
\theta_{, \tau} & =\left[\Delta_{1} \theta_{, x x}+\Delta_{2} \theta_{, z z}+\Delta_{3}\left(-2 \theta_{, x z}-\theta_{, x}^{2}+\theta_{, z}^{2}\right)\right. \\
& \left.+\Delta_{4} \theta_{, x} \theta_{, z}\right]+\frac{1}{2} \bar{E}^{2}(z) \sin 2(\alpha-\theta)+\frac{1}{2}\left(\psi_{, x x}+\psi_{, z z}\right) \\
& -\psi_{, z} \theta_{, z}+\psi_{, x} \theta_{, x}+\gamma\left[\sin 2 \theta \psi_{, x z}+\frac{1}{2}\left(\psi_{, x x}-\psi_{, z z}\right)\right],
\end{aligned}
$$

где $\Delta_{1}=\sin ^{2} \theta+K_{31} \cos ^{2} \theta, \quad \Delta_{2}=\cos ^{2} \theta+K_{31} \sin ^{2} \theta$, $\Delta_{3}=\frac{\left(1-K_{31}\right)}{2} \sin 2 \theta, \quad \Delta_{4}=\left(K_{31}-1\right) \cos 2 \theta, \quad \gamma=\gamma_{2} / \gamma_{1}$, $K_{31}=K_{3} / K_{1}, \quad$ а $\quad K_{1} \quad$ и $\quad K_{3} \quad$ - коэффициенты упругости, соответствующие изгибным и продольным деформациям. Безразмерное уравнение Навье-Стокса, записанное с помощью функции тока $\psi$, имеет вид [10]

$$
\delta_{2}\left[(\Delta \psi)_{, \tau}+\psi_{, z}(\Delta \psi)_{, x}-\psi_{, x}(\Delta \psi)_{, z}\right]=\hat{\mathscr{L}} \psi+\mathscr{F},
$$

где $\Delta \psi=\psi_{, x x}+\psi_{, z z}$, а оба оператора $\hat{\mathscr{L}}$ и $\mathscr{F}$ приведены в Приложении. Здесь

$$
\delta_{1}=\frac{4 K_{1}}{\epsilon_{0} \epsilon_{a} U^{2}}, \quad \delta_{2}=\frac{\rho \epsilon_{0} \epsilon_{a}}{4 \gamma_{1}^{2}} U^{2}
$$

- два параметра ЖК-системы, а функция

$$
\bar{E}(z)=\frac{2 d}{U} \sin \alpha E(z)
$$

описывает безразмерное электрическое поле. В свою очередь, безразмерное электрическое поле удовлетворяет основному уравнению электростатики для диэлектриков

$$
\frac{\partial}{\partial z}\left[\left(\frac{\epsilon_{\perp}}{\epsilon_{a}}+\sin \theta\right) \bar{E}(z)\right]=0, \quad \int_{-1}^{1} \bar{E}(z) d z=1,
$$

где $\epsilon_{\perp}-$ величина диэлектрической проницаемости ЖК-материала в направлении, перпендикулярном полю директора $\hat{\mathbf{n}}$.
Мы будем рассматривать ЖК-канал с размерами $L / d=10$, помещенный между двумя электродами таким образом, что вектор $\mathbf{E}$ направлен под углом $\alpha$ к орту $\hat{\mathbf{i}}$. Будем изучать случай жесткого сцепления, когда граничные условия для угла $\theta$ могут быть записаны в виде

$$
\theta_{-10<x<10, z= \pm 1}=\theta_{x= \pm 10,-1<z<1}=0 .
$$

Поле скорости $\mathbf{v}$ подчиняется условию прилипания на твердых ограничивающих поверхностях ЖК-канала и может быть записано с помощью безразмерной функции тока как

$$
\begin{aligned}
& \left(\psi_{, z}\right)_{-10<x<10, z= \pm 1}=\left(\psi_{, z}\right)_{x= \pm 10,-1<z<1}=0, \\
& \left(\psi_{, x}\right)_{-10<x<10, z= \pm 1}=\left(\psi_{, x}\right)_{x= \pm 10,-1<z<1}=0 .
\end{aligned}
$$

Уравнения (1)-(3) необходимо дополнить начальными условиями как для поля директора, так и для поля скорости. Начальное условие для угла $\theta$ мы выберем в виде

$$
\theta(x, z, 0)=\theta_{0} \cos \theta\left(q_{z} z\right) \cos \theta\left(q_{x} x\right)
$$

где $\theta_{0}$ - амплитуда, а $q_{x}$ и $q_{z}$ - волновые числа соответствующей Фурье-моды. В нашем случае волновые числа $q_{x}$ и $q_{z}$ соответствующей Фурье-моды имеют вид [7]

$$
\begin{aligned}
& q_{x}=\frac{\pi}{20}(2 k+1), k=0,1,2, \ldots, \\
& q_{z}=\frac{\pi}{20}(2 l+1), l=0,1,2, \ldots .
\end{aligned}
$$

В свою очередь, начальное условие для скорости $v(x, z, 0)=0$, записанное с помощью функции тока, принимает вид

$$
\psi(x, z, 0)=0
$$

Следует отметить, что в процессе переориентации поля директора под действием сильного электрического поля баланс импульсов и угловых моментов, действующих на единицу ЖК-объема, разворачивает поле директора к его равновесному распределению $\hat{\mathbf{n}}_{e q}$ по всему объему ЖК-фазы, которое описывается углом $\theta_{e q}(x, z)$. Время, необходимое для переориентации поля директора в положение $\theta_{e q}(x, z)$, есть время релаксации $\tau_{R}$ системы. Его величина зависит от величины электрического поля $\bar{E}$ и углов $\alpha$ и $\theta_{0}$. В свою очередь, безразмерные волновые числа $q_{x}$ и $q_{z}$ определяются из условия минимума полной энергии $W=W_{\text {elast }}+W_{\text {el }}$, где

$$
\begin{aligned}
\frac{2}{\delta_{1}} W_{\text {elast }} & =\int d x \int d z\left[\left(\left(\theta_{e q}\right)_{, x}^{2}+\left(\theta_{e q}\right)_{, z}^{2}\right)\right. \\
& \left.\times\left(\sin ^{2} \theta_{e q}+K_{31} \cos ^{2} \theta_{e q}\right)\right] \\
& +\int d x \int d z\left(K_{31}-1\right) \sin 2 \theta_{e q}\left(\theta_{e q}\right)_{, x}\left(\theta_{e q}\right)_{, z}
\end{aligned}
$$


— вклад упругих сил, а

$$
W_{\mathrm{el}}=-\int d x \int d z E\left(\theta_{e q}\right) \cos ^{2}\left(\theta_{e q}-\alpha\right)
$$

- вклад электрических сил в общую энергию $W$ соответственно.

Таким образом, система уравнений (1)-(3), (7) и (9), (10), дополненная граничными (4), (5) и начальными условиями (6) и (8), образует самосогласованную систему нелинейных дифференциальных уравнений в частных производных, описывающих эволюцию как поля директора $\hat{\mathbf{n}}$, так и поля скорости $\mathbf{v}$ к их равновесным распределениям по всему объему микрометрового ЖК-канала под действием сильного электрического поля $\mathbf{E}$, направленного под углом $\alpha$ к горизонтальным ограничивающим поверхностям.

Располагая значениями угла $\theta(x, z, \tau)$ в процессе его релаксации к равновесному распределению по всему ЖК-каналу, мы можем также расчитать не только эволюцию угловой скорости $\boldsymbol{\omega}$ поля директора $\hat{\mathbf{n}}$

$$
\omega=\hat{\mathbf{n}} \times \dot{\hat{\mathbf{n}}}=-\dot{\theta}(x, z, \tau) \hat{\mathbf{j}}=-\omega(x, z, \tau) \hat{\mathbf{j}},
$$

но и эволюцию компонент тензора напряжения (ТН)

$$
\begin{gathered}
\sigma_{x x}=\frac{1}{4}\left(E^{2}-\mathscr{A}\right) \sin 2 \theta, \\
\sigma_{z z}=\sigma_{x x}+\frac{1}{2} \mathscr{B} \sin 2 \theta+\left(\frac{\epsilon_{\perp}}{\epsilon_{a}}+\sin ^{2} \theta\right) E^{2}, \\
\sigma_{x z}=\frac{1}{4}\left(-E^{2}+\mathscr{A}\right) \sin 2 \theta, \\
\sigma_{z x}=\frac{1}{4}\left(E^{2}+\mathscr{A}\right) \sin 2 \theta,
\end{gathered}
$$

где $\mathscr{A}=\frac{\gamma_{2}}{\gamma_{1}} \cos 2 \theta E^{2}$ и $\mathscr{B}=\frac{\gamma_{2}}{\gamma_{1}} \sin 2 \theta E^{2}-$ безразмерные функции. В нашем случае безразмерные компоненты ТН принимают вид

$$
\sigma_{i j}=\mathscr{P} \delta_{i j}+\sigma_{i j}^{\mathrm{el}}+\sigma_{i j}^{\mathrm{vis}}+\delta_{1} \sigma_{i j}^{\mathrm{elast}},
$$

где $\mathscr{P}$ - гидростатическое давление в ЖК-канале, а $\sigma_{i j}^{\text {el }}, \sigma_{i j}^{\text {vis }}$ и $\sigma_{i j}^{\text {elast }}-$ безразмерные компоненты ТН, соответствующие электрическим, вязким и упругим силам соответственно.

Для случая нематика, образованного молекулами 5ЦБ, при температуре $300 \mathrm{~K}$ и плотности $10^{3} \mathrm{~kg} / \mathrm{m}^{3}$, а также величине напряжения в $U=200 \mathrm{~V}$, приложенного поперек ЖК-канала толщиной в $200 \mu \mathrm{m}$, значения параметров, которые входят в вышеописанные уравнения, равны: $\delta_{1}=8.6 \cdot 10^{-6}, \delta_{2}=0.19, \gamma=-1.1$ и $K_{31}=1.17$. Следует отметить, что величина порогового напряжения в нашем случае равна $E_{\text {th }} \sim 1.05 \times 10^{4} \mathrm{~V} / \mathrm{m}$, так что $E \sim 100 E_{\mathrm{th}}$, а толщина и величина напряжения, приложенного поперек ЖК-канала, соответствовали данным, использованным при исследовании эволюции ЯМР-спектров в ЖК-ячейках, образованных молекулами дейтерированного 4-n-пентил-4'-цианобифенила [6].
Принимая во внимание тот факт, что $\delta_{1} \ll 1$, упругим вкладом в ТН можно пренебречь, в то время как вязкий вклад в ТН $\sigma_{i j}$ может быть переписан в виде

$$
\begin{gathered}
\sigma_{x x}^{\mathrm{vis}}=-\frac{1}{4} \mathscr{B} \sin 2 \theta, \\
\sigma_{x z}^{\mathrm{vis}}=\frac{1}{4}\left(-E^{2}+\mathscr{A}\right) \sin 2 \theta, \\
\sigma_{z x}^{\mathrm{vis}}=\frac{1}{4}\left(E^{2}+\mathscr{A}\right) \sin 2 \theta,
\end{gathered}
$$

и

$$
\sigma_{z z}^{\mathrm{vis}}=-\sigma_{x x}^{\mathrm{vis}} .
$$

В нашем случае, когда электрическое поле $\mathbf{E}$ приложено поперек ЖК-канала, вклад электрических сил в общий ТН $\sigma_{i j}^{\text {el }}=\frac{1}{2}\left(E_{i} D_{j}+D_{i} E_{j}\right)$ имеет только одну компоненту

$$
\sigma_{z z}^{\mathrm{el}}=\left(\frac{\epsilon_{\perp}}{\epsilon_{a}}+\sin ^{2} \theta\right) E^{2},
$$

где D - вектор электрической индукции. C другой стороны, в нашем случае гидродинамическое давление имеет вид

$$
\mathscr{P}=\frac{1}{4}\left(\mathscr{B}-\mathscr{A}+E^{2}\right) \sin 2 \theta,
$$

поскольку оно удовлетворяет уравнению

$$
\sigma_{x x, x}+\sigma_{x z, x}+\mathscr{P}_{, x}=0 .
$$

\section{3. Эволюция поля директора, скорости и компонент ТН в ЖК-канале под действием сильного электрического поля}

Когда сильное электрическое поле $\mathbf{E}=E \hat{\mathbf{k}}\left(E \sim 100 E_{\mathrm{th}}\right)$ (в положительном смысле) включено в момент времени $\tau=0$, под углом $\alpha\left(\sim \frac{\pi}{2}\right)$ к горизонтальным ограничивающим поверхностям ЖК-канала, планарно и однородно ориентированный нематический образец, образованный молекулами 5ЦБ, стремится переориентироваться в направлении вектора Е. Этот процесс переориентации описывается углом $\theta(x, z, \tau)$, а инициируемое разворотом директора $\hat{\mathbf{n}}$ поле скорости $\mathbf{v}=u(x, z, \tau) \hat{\mathbf{i}}+w(x, z, \tau) \hat{\mathbf{k}}$ описывается системой нелинейных дифференциальных уравнений в частных производных (1)-(3), (9) и (10), дополненной граничными (4), (5) и начальными (6) и (8) условиями, как для угла $\theta$, так и для функции тока $\psi$, при том, что значения волновых чисел $q_{x}$ и $q_{z}$ определяются из условия минимума полной энергии $W=W_{\text {elast }}+W_{\text {el }}$. Для случая нематика, образованного молекулами 5ЦБ, при температуре $300 \mathrm{~K}$ и плотности $10^{3} \mathrm{~kg} / \mathrm{m}^{3}$, величина порогового напряжения равна $E_{\text {th }} \sim 1.05 \cdot 10^{4} \mathrm{~V} / \mathrm{m}$, так что $E \sim 100 E_{\mathrm{th}}$. Наш предыдущий анализ подобных систем показал, что при определенном балансе упругих, вязких и электрических моментов и импульсов, действующих на единицу объема ЖК-фазы, существует 


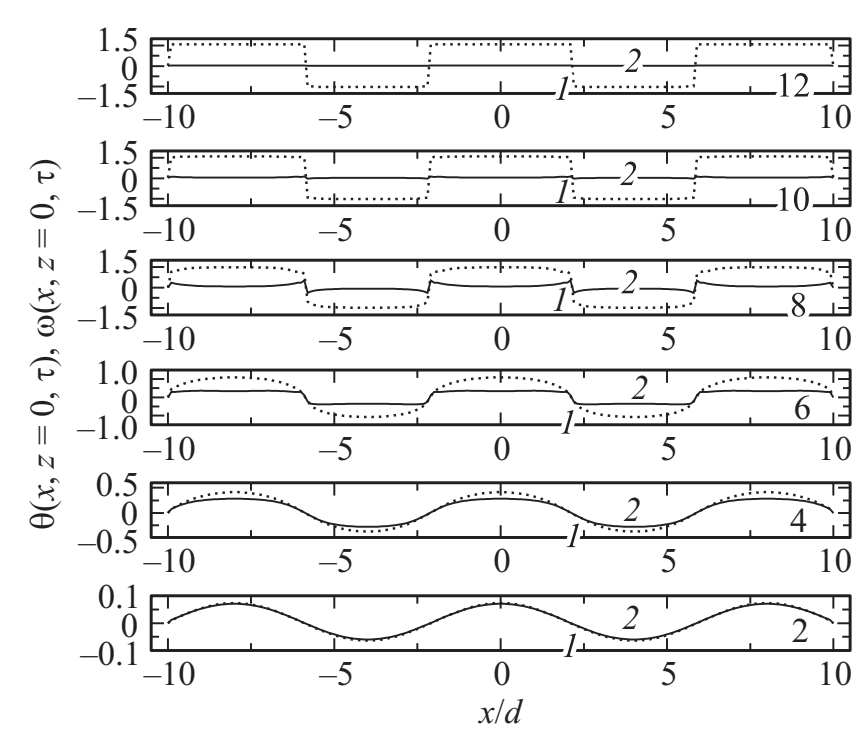

Рис. 3. Эволюция распределения угла $\theta(x, z=0, \tau)$ (кривые 1) и угловой скорости $\omega(x, z=0, \tau)$ (кривые 2) вдоль оси $x \in[-10,10]$ к их равновесным распределениям $\theta_{e q}\left(x, z=0, \tau=\tau_{R}(\right.$ in $\left.)=12\right)$ и $\omega_{e q}\left(x, z=0, \tau=\tau_{R}(\right.$ in $\left.)=12\right)$ соответственно, под действием сильного электрического поля $\mathbf{E}=E \hat{\mathbf{k}} \quad(E \sim 1.05 \mathrm{~V} / \mu \mathrm{m})$, направленного под углом $\alpha=1.57\left(\sim 89.96^{\circ}\right)$ к горизонтальным ограничивающим поверхностям ЖК-канала. Промежуточные состояния соответствуют временам $\tau=2(\sim 12 \mathrm{~ms}), 4(\sim 24 \mathrm{~ms}), 6(\sim 36 \mathrm{~ms})$, $8(\sim 48 \mathrm{~ms})$ и $12(\sim 72 \mathrm{~ms})$ соответственно.

пороговое значение амплитуды угла $\theta_{0}^{\text {th }}$, выше которого характер переориентации поля директора претерпевает как качественное, так и количественное изменение [10]. Так, было показано, что при всех прочих равных условиях, при $\theta<\theta_{0}^{\text {th }}$, поле директора $\hat{\mathbf{n}}(x, z, \tau)$ разворачивается в направлении вектора $\mathbf{E}\left(|\mathbf{E}| \sim 100 E_{\mathrm{th}}\right)$ как единое целое, т. е. как монодомен. В то же время при значениях $\theta \geq \theta_{0}^{\text {th }}$ переориентация поля директора характеризуется формированием квазипериодических структур по всему объему, занимаемому ЖК-фазой [10]. На рис. 3 (кривые 1, точечные линии) представлены результаты расчета эволюции угла $\theta(x, z=0, \tau)$ к его равновесному распределению $\theta_{e q}(x, z=0)$ вдоль оси $x \in[-10,10]$, которое достигается спустя $\tau_{R}($ in $)=12(\sim 72 \mathrm{~ms})$ единиц безразмерного времени. Эти расчеты были получены для случая жесткого сцепления ЖК-молекул с ограничивающими поверхностями, при значениях углов $\alpha=1.57\left(\sim 89.96^{\circ}\right)$ и $\theta_{0}=0.01\left(\sim 1.1^{\circ}\right)$, а критерий сходимости итерационной процедуры был выбран равным $\epsilon=\left|\left(\theta_{(m+1)}-\theta_{(m)}\right) / \theta_{(m)}\right| \sim 10^{-4}$ и итерационная процедура продолжалась до достижения заданной точности [11]. Промежуточные состояния соответствуют временам $\tau=2(\sim 12 \mathrm{~ms}), 4(\sim 24 \mathrm{~ms}), 6(\sim 36 \mathrm{~ms})$, $8(\sim 48 \mathrm{~ms})$ и $12(\sim 72 \mathrm{~ms})$ соответственно. Здесь безразмерное время $\tau=\frac{\epsilon_{0} \epsilon_{a}}{\gamma_{1}}\left(\frac{U}{2 d}\right)^{2} t$ отсчитывалось с момента включения электрического поля. В этом случае равновесное распределение угла $\theta_{e q}(x, z=0)$ вдоль оси $x \in[-10,10]$ характеризуется отчетливо выраженной квазипериодической структурой с узлами в точках $x= \pm 2.175$ и \pm 5.83 . Отметим, что величины волновых чисел $q_{x}$ и $q_{z}$, которые обеспечивают минимум энергии $W_{\text {elast }}+W_{\text {el }}$, равны $q_{x}=0.785$ и $q_{z}=64.336$. Эволюция распределения безразмерной угловой скорости $\omega(x, z=0, \tau)$ к ее равновесному распределению $\omega_{e q}\left(x, z=0, \tau=\tau_{R}(\right.$ in $\left.)\right)=0$, вдоль оси $x \in[-10,10]$, представлена на рис. 3 (кривые 2, сплошные линии). Промежуточные состояния соответствуют безразмерным временам $\tau=2(\sim 12 \mathrm{~ms}), \tau=4(\sim 24 \mathrm{~ms})$, $\tau=6(\sim 36 \mathrm{~ms}), \tau=8(\sim 48 \mathrm{~ms}), \tau=10 \quad(\sim 60 \mathrm{~ms})$, и $\tau=\tau_{R}($ in $)=12(\sim 72 \mathrm{~ms})$ соответственно. В течение первых 4 единиц безразмерного времени $(\sim 24 \mathrm{~ms})$ распределение угловой скорости $\omega(x, z=0, \tau)$ практически следует за распределением угла $\theta(x, z=0, \tau)$. При этом отчетливо видно, как начинают формироваться в ЖК-канале несколько вихрей, причем положения узлов решетки задают границы формируемых вихрей. Так, согласно нашим расчетам, представленным на рис. 3 (кривые 2), отчетливо наблюдаются три вихря $(-10<x<-5.83,-2.175<x<2.175$ и $5.83<x<10)$, вращающихся по часовой стрелке, и два вихря $(-5.83<x<-2.175$ и $2.175<x<5.83)$, вращающихся против часовой стрелки. Спустя время $\tau=10(\sim 60 \mathrm{~ms})$ вращение вихрей практически полностью прекратилось и установилось равновесное распределение поля директора вдоль оси $x \in[-10,10]$, характеризующееся отчетливо выраженной квазипериодической структурой с узлами в точках $x= \pm 2.175$ и \pm 5.83 .

Теперь, располагая распределением угла $\theta(x, z, \tau)$, мы можем, используя уравнение (12), расчитать безразмерные компоненты ТН

$$
\sigma_{i j}(x, z, \tau)=\left(\frac{4 d^{2}}{\epsilon_{0} \epsilon_{a} U^{2}}\right) \bar{\sigma}_{i j}(x, z, \tau),
$$

где $\bar{\sigma}_{i j}(x, z, \tau)$ - размерные значения тензора напряжения. Эволюция безразмерных сдвиговых компонент ТН $\sigma_{x z}(x, z=0, \tau)$ и $\sigma_{z x}(x, z=0, \tau)$ (рис. 4, $\left.a, b\right)$ и нормальной компоненты $\sigma_{x x}(x, z=0, \tau)$ (рис. $\left.5, b\right)$ к их равновесным значениям в шести точках, отстоящих на расстояниях $x=0.5$ (кривая 1), 1.6 (кривая 2), 2.16 (кривая 3), 2.18 (кривая 4), 2.32 (кривая 5) и 3.42 (кривая 6) от центра ЖК-канала, в течение первых 20 единиц безразмерного времени показаны на рис. 4 и 5. Расчеты свидетельствуют о том, что абсолютные величины двух сдвиговых компонент ТН $\left|\sigma_{z x}\right|$ и $\left|\sigma_{x z}\right|$, а также нормальной компоненты $\left|\sigma_{x x}\right|$ на начальном этапе эволюции, соответствующем временам $\tau \sim 4 \div 6(\sim 24 \div 36 \mathrm{~ms})$, достигают максимальных значений $\sim 0.3(\sim 6.6 \mathrm{~Pa})$, а затем быстро убывают к нулю. В свою очередь, безразмерная нормальная компонента ТН $\sigma_{z z}(x, z=0, \tau)$ (рис. 5, $a$ ) на начальном этапе эволюции, соответствующем временам $\tau \sim 4 \div 7(\sim 24 \div 42 \mathrm{~ms})$, осциллирует около значения $\sim 0.5(\sim 10 \mathrm{~Pa})$, для всех вышеуказанных значений расстояния вдоль оси $x$, а затем 

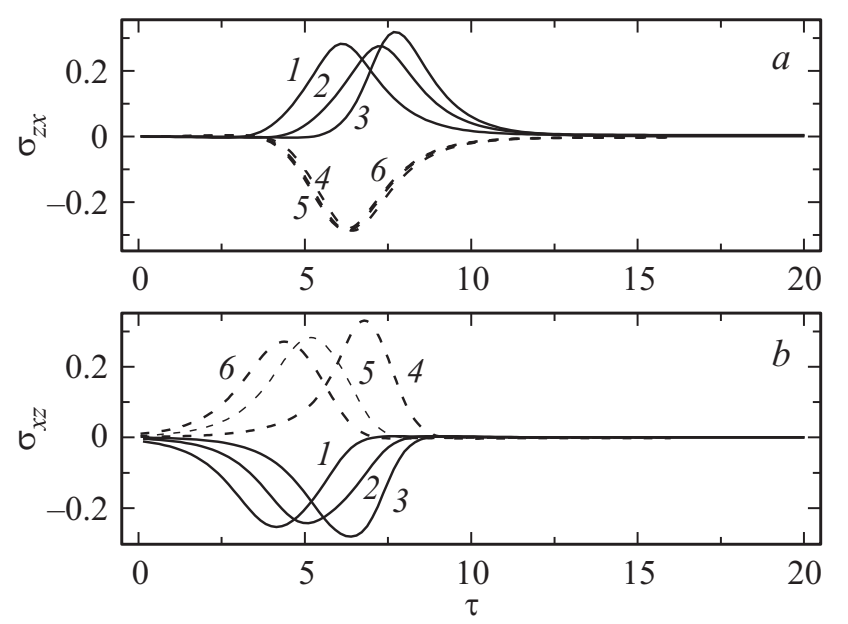

Рис. 4. Эволюция сдвиговых компонент ТН $\sigma_{z x}(x, z=0, \tau)(a)$ и $\sigma_{x z}(x, z=0, \tau)(b)$ в точках $x=0.5$ (кривые 1), $x=1.6$ (кривые 2), $x=2.16$ (кривые 3), $x=2.18$ (кривые 4), $x=2.32$ (кривые 5) и $x=3.42$ (кривые 6) соответственно к их равновесным распределениям $\sigma_{z x}^{e q}$ и $\sigma_{x z}^{e q}$ под действием сильного электрического поля $\mathbf{E}=E \hat{\mathbf{k}} \quad(E \sim 1.05 \mathrm{~V} / \mu \mathrm{m}), \quad$ направленного под углом $\alpha=1.57 \quad\left(\sim 89.96^{\circ}\right) \quad$ к горизонтальным ограничивающим поверхностям ЖК-канала.
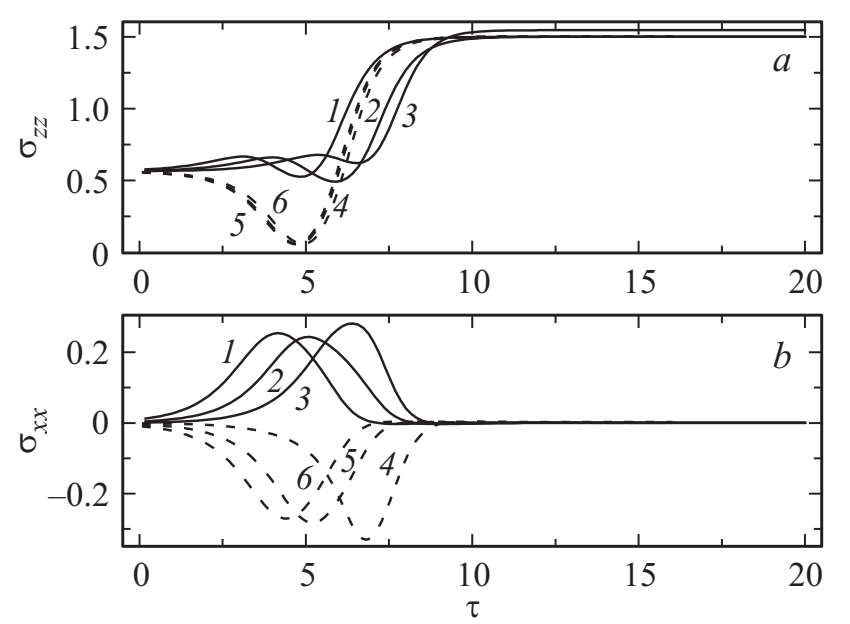

Рис. 5. То же, что на рис. 4, только для нормальных компонент ТН $\sigma_{z z}(x, z=0, \tau)(a)$ и $\sigma_{x x}(x, z=0, \tau)(b)$ соответственно.

монотонно возрастает и на конечном этапе эволюции давление растяжения $\sigma_{z z}$, обусловленное сильным электрическим полем $\mathbf{E}=E \hat{\mathbf{k}}(E \sim 1.05 \mathrm{~V} / \mu \mathrm{m})$, достигает величины $\sim 1.5(\sim 33 \mathrm{~Pa})$. Следует отметить, что вблизи узла $x=2.175$, в точках $x=2.16$ (кривые 3 ) и $x=2.18$ (кривые 4), как сдвиговые компоненты ТН $\sigma_{x z}(x, z=0, \tau)$ (рис. $\left.4, b\right)$ и $\sigma_{z x}(x, z=0, \tau)$ (рис. $\left.4, a\right)$, так и нормальная компонента $\sigma_{x x}(x, z=0, \tau)$ (рис. $\left.5, b\right)$ меняют знак, что свидетельствует о том, что в окрестности узла $x=2.175$ возникли разнонаправленные вихревые течения, инициируемые сильным электрическим полем $E \sim 100 E_{\mathrm{th}}$.
Предположим далее, что в момент времени $\tau=20$ $(\sim 0.12 \mathrm{~s})$ электрическое поле будет выключено, т.е. $E=0$. В этом случае поле директора $\hat{\mathbf{n}}(x, z, \tau)$ под действием вязких, упругих и поверхностных сил и моментов, действующих на единицу объема ЖК-фазы, начинает переориентироваться из состояния, характеризующегося углом $\theta_{e q}(x, z)$, в состояние, характеризующееся планарной ориентацией ЖК-канала. При этом угол $\theta(x, z, \tau)$ должен стремиться к нулю. Следует отметить, что время релаксации $\tau_{R}(\mathrm{off})$, в связи с малостью вязких, упругих и поверхностных сил и моментов, по сравнению с электрическими, значительно больше времен $\tau_{R}($ in). На рис. 6 (кривые 1) представлены результаты расчетов эволюций угла $\theta(x, z=0, \tau)$ вдоль оси $x \in[-10,10]$ для случая жесткого сцепления ЖК-молекул с ограничивающими поверхностями для следующих, после выключения электрического поля, 20 единиц безразмерного времени. Здесь представлены состояния, соответствующие временам $\tau=22(\sim 0.132 \mathrm{~s}), 24(\sim 0.144 \mathrm{~s}), 26(\sim 0.156 \mathrm{~s})$, $30(\sim 0.18 \mathrm{~s}), 35(\sim 0.21 \mathrm{~s})$, и $\tau=40(\sim 0.24 \mathrm{~s})$. Результаты расчетов указывают на то, что быстрее релаксируют области, удаленные от положений узлов квазипериодической структуры, т.е. вблизи точек $x= \pm 2.175$ и \pm 5.83 . Безразмерное время релаксации $\tau_{R}($ off $)$ поля директора к планарно ориентированному распределению по всему объему ЖК-канала равно 400, или $\sim 2.4 \mathrm{~s}$. Эволюция распределения безразмерной угловой скорости $\omega(x, z=0, \tau)$ к ее равновесному распределению

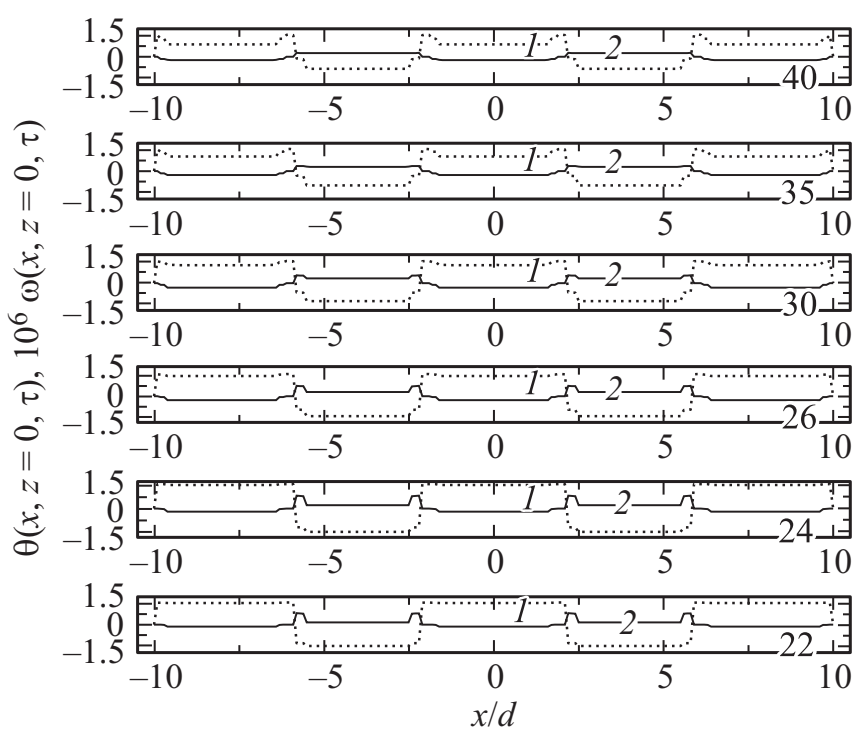

Рис. 6. Эволюция распределения угла $\theta(x, z=0, \tau)$ (кривые 1) и угловой скорости $\omega(x, z=0, \tau)$ (кривые 2) вдоль оси $x \in[-10,10]$ к их равновесным распределениям $\theta_{e q}\left(x, z=0, \tau=\tau_{R}(\mathrm{off})\right)$ и $\omega_{e q}\left(x, z=0, \tau=\tau_{R}(\mathrm{off})\right)$ соответственно, с момента отключения электрического поля $\mathbf{E}=0$. Промежуточные состояния соответствуют временам $\tau=22$ $(\sim 0.132 \mathrm{~s}), 24 \quad(\sim 0.144 \mathrm{~ms}), 26(\sim 0.156 \mathrm{~s}), 30(\sim 0.18 \mathrm{~s})$, $35(\sim 0.21 \mathrm{~s})$ и $40(\sim 0.24 \mathrm{~s})$ соответственно. 
$\omega_{e q}\left(x, z=0, \tau=\tau_{R}(\mathrm{off})\right)=0$, вдоль оси $x \in[-10,10]$, в течение следующих 20 единиц времени после выключения электрического поля показана на рис. 6 (кривые 2). Представлены состояния, соответствующие тем же безразмерным временам, что и для угла $\theta\left(x, z=0, \tau=\tau_{R}(\mathrm{off})\right)$. Следует отметить, что в течение первых 6 единиц безразмерного времени после выключения электрического поля абсолютная величина угловой скорости $|\omega(x, z, \tau)|$ достигает незначительного максимального значения вблизи узлов квазипериодической структуры в точках $x= \pm 2.175$ и \pm 5.83 , а затем, в течение следующих 14 единиц безразмерного времени, быстро релаксирует к нулю. При этом абсолютная величина угловой скорости $\omega(\mathrm{off})$ примерно на 6 порядков меньше абсолютной величины $\omega$ (in).

Наконец, рассмотрим третий случай, когда сильное электрическое поле $\mathbf{E}=-E \hat{\mathbf{k}}(E \sim 1.05 \mathrm{~V} / \mu \mathrm{m})$ включено в момент времени $\tau=248$ (случай $A$ ) или 250 (случай $B$ ) (в отрицательном смысле), под углом $\alpha\left(\sim-\frac{\pi}{2}\right)$ к горизонтальным ограничивающим поверхностям ЖК-канала. Следует отметить, что в обоих случаях $A$ и $B$ отсчет времени начался с момента включения электрического поля $\mathbf{E}=E \hat{\mathbf{k}}$. Этот процесс переориентации поля директора из положения $\hat{\mathbf{n}}^{\text {off }}$ в направлении вектора $\mathbf{E}$ описывается углом $\theta(x, z, \tau)$. Здесь $\hat{\mathbf{n}}^{\text {off }}$ - ориентация поля директора спустя время $\tau$ (off), т.е. ориентация, соответствующая планарно и однородно ориентированному нематическому образцу. Как и в случае $\mathbf{E}=E \hat{\mathbf{k}}$, процесс переориентации $\hat{\mathbf{n}}$ описывается системой нелинейных дифференциальных уравнений в частных производных (1)-(3), (9) и (10), дополненной граничными (4), (5) и начальными (6) и (8) условиями, как для угла $\theta$, так и для функции тока $\psi$, при том, что значения волновых чисел $q_{x}$ и $q_{z}$ определяются из условия минимума полной энергии $W=W_{\text {elast }}+W_{\mathrm{el}}$. Выбор двух времен, $\tau=248$ (случай $A$ ) или 250 (случай $B)$, включения электрического поля $\mathbf{E}=-E \hat{\mathbf{k}}$ влияет на величину порогового значения амплитуды угла $\theta_{0}^{\text {th }}$ и, тем самым, на характер переориентации поля директора под действием сильного электрического поля. Так, в случае $A(\tau=248)$ электрическое поле $\mathbf{E}$ было выключено в течение $\tau($ off $)=248-20=228$ единиц безразмерного времени. Таким образом, в случае $A$ начальное условие для угла $\theta$ принимает вид

$$
\theta(x, z, 0)=\theta^{\text {off }}(x, z),
$$

а значение угла $\alpha=-1.57$.

На рис. 7 (кривые 1 , точечные линии) представлены результаты расчета эволюции угла $\theta(x, z=0, \tau)$ к его равновесному распределению $\theta_{e q}(x, z=0)$ вдоль оси $x \in[-10,10]$, которое достигается спустя $\tau_{R}($ in $)=10$ $(\sim 60 \mathrm{~ms})$ единиц безразмерного времени после повторного включения электрического поля $\mathbf{E}=-E \hat{\mathbf{k}}$ $(E \sim 1.05 \mathrm{~V} / \mu \mathrm{m})$. Эти расчеты были получены для случая жесткого сцепления ЖК-молекул с ограничивающими поверхностями, при значении угла $\alpha=-1.57$

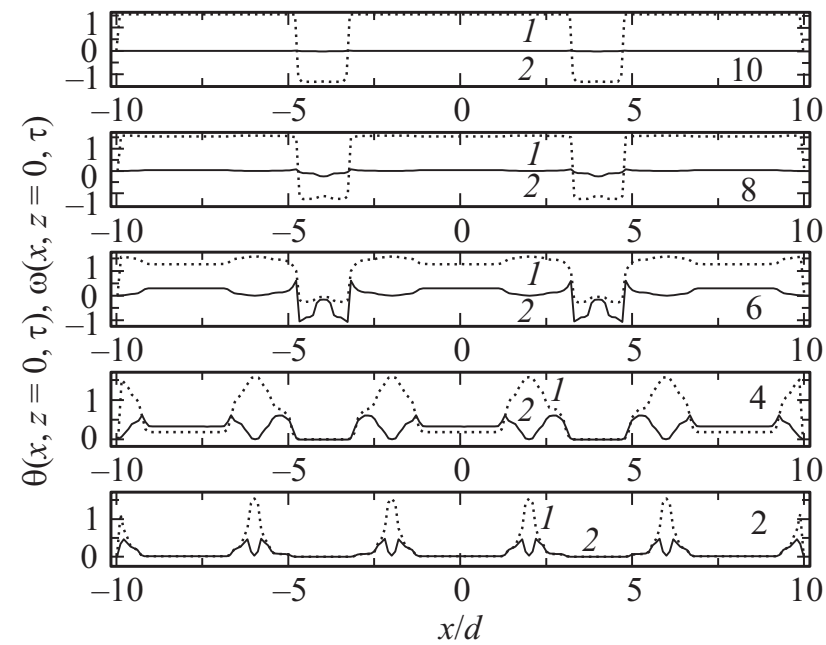

Рис. 7. Эволюция распределения угла $\theta(x, z=0, \tau)$ (кривые 1) и угловой скорости $\omega(x, z=0, \tau)$ (кривые 2) вдоль оси $x \in[-10,10]$ к их равновесным распределениям $\theta_{e q}\left(x, z=0, \tau=\tau_{R}(\right.$ in $\left.)=10\right)$ и $\omega_{e q}\left(x, z=0, \tau=\tau_{R}(\right.$ in $\left.)=10\right)$ соответственно (случай $A$ ), под действием сильного электрического поля $\mathbf{E}=-E \hat{\mathbf{k}}(E \sim 1.05 \mathrm{~V} / \mu \mathrm{m})$, направленного под углом $\alpha=-1.57\left(\sim-89.96^{\circ}\right)$ к горизонтальным ограничивающим поверхностям ЖК-канала. Промежуточные состояния соответствуют временам $\tau=2(\sim 12 \mathrm{~ms}), 4$ ( 24 ms), $6(\sim 36 \mathrm{~ms}), 8(\sim 48 \mathrm{~ms})$ и $10(\sim 60 \mathrm{~ms})$ после повторного включения электрического поля.

$\left(\sim-89.96^{\circ}\right)$. Промежуточные состояния соответствуют временам $\tau=2(\sim 12 \mathrm{~ms}), 4$ ( 24 ms), 6 ( $36 \mathrm{~ms})$, $8(\sim 48 \mathrm{~ms})$ и $10(\sim 60 \mathrm{~ms})$ соответственно. Здесь безразмерное время $\tau=\frac{\epsilon_{0} \epsilon_{a}}{\gamma_{1}}\left(\frac{U}{2 d}\right)^{2} t$ отсчитывалось с момента повторного включения электрического поля. В этом случае равновесное распределение угла $\theta_{e q}(x, z=0)$ вдоль оси $x \in[-10,10]$ характеризуется отчетливо выраженной квазипериодической структурой с узлами в точках $x= \pm 3.26$ и \pm 4.72 . Эволюция распределения безразмерной угловой скорости $\omega(x, z=0, \tau)$ к ее равновесному распределению $\omega_{e q}\left(x, z=0, \tau=\tau_{R}(\right.$ in $\left.)\right)=0$, вдоль оси $x \in[-10,10]$, представлена на рис. 7 (кривые 2, сплошные линии). Промежуточные состояния соответствуют безразмерным временам $\tau=2(\sim 12 \mathrm{~ms})$, $\tau=4 \quad(\sim 24 \mathrm{~ms}), \quad \tau=6 \quad(\sim 36 \mathrm{~ms}), \quad \tau=8 \quad(\sim 48 \mathrm{~ms})$ и $\tau=\tau_{R}($ in $)=10(\sim 60 \mathrm{~ms})$ соответственно. При этом отчетливо видно, как спустя 6 единиц безразмерного времени начинает формироваться в ЖК-канале несколько вихрей, причем положения узлов решетки задают границы формируемых вихрей. Так, согласно нашим расчетам, представленным на рис. 7 (кривые 2), отчетливо наблюдаются три вихря $(-10<x<-4.72$, $-3.26<x<3.26$ и $4.72<x<10$ ), вращающихся по часовой стрелке, и два вихря $(-4.72<x<-3.26$ и $3.26<x<4.72)$, вращающихся против часовой стрелки. Спустя время $\tau_{R}(\mathrm{in})=10(\sim 60 \mathrm{~ms})$ вращение вихрей практически полностью прекратилось и установилось равновесное распределение поля директора вдоль оси 


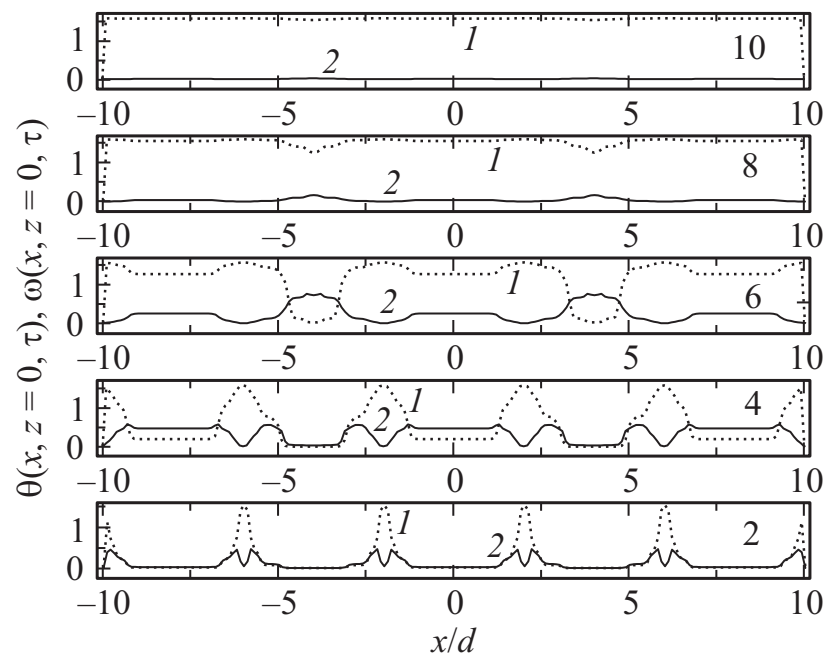

Рис. 8. То же, что на рис. 7 , только для случая $B$.

$x \in[-10,10]$, характеризующееся отчетливо выраженной квазипериодической структурой с узлами в точках $x= \pm 3.26$ и \pm 4.72 .

Иначе происходит процесс переориентации поля директора в случае В, когда директор переориентируется из положения $\hat{\mathbf{n}}^{\text {off }}$ в направлении вектора $\mathbf{E}$, описываемого углом $\theta(x, z, \tau)$, под действием сильного электрического поля $\mathbf{E}=-E \hat{\mathbf{k}}(E \sim 1.05 \mathrm{~V} / \mu \mathrm{m})$, включенного в момент времени $\tau=250$, под углом $\alpha\left(\sim-\frac{\pi}{2}\right)$ к горизонтальным ограничивающим поверхностям ЖК-канала. В этом случае электрическое поле $\mathbf{E}$ было выключено в течение $\tau$ (off) $=250-20=230$ единиц безразмерного времени и начальное условие для угла $\theta$ определялось другой функцией $\theta^{\text {off }}(x, z)$, которой соответствовала меньшая величина амплидуды $\theta_{0}^{\text {off }}$ (см. уравнение (6)).

На рис. 8 (кривые 1 , точечные линии, и 2, сплошные линии) представлены результаты расчета эволюции угла $\theta(x, z=0, \tau)$ и безразмерной угловой скорости $\omega(x, z=0, \tau)$ к их равновесным распределениям $\theta_{e q}(x, z=0)$ и $\omega_{e q}(x, z=0)$ вдоль оси $x \in[-10,10]$ соответственно. Эти расчеты были получены для случая жесткого сцепления ЖК-молекул с ограничивающими поверхностями, при значении угла $\alpha=-1.57\left(\sim-89.96^{\circ}\right)$. Промежуточные состояния соответствуют временам $\tau=2(\sim 12 \mathrm{~ms}), 4(\sim 24 \mathrm{~ms})$, 6 ( $\sim 36 \mathrm{~ms}), 8(\sim 48 \mathrm{~ms})$ и $10(\sim 60 \mathrm{~ms})$ соответственно. Следует отметить, что в обоих выше описанных случаях $A$ и $B$, в течение первых 4еддинц безразмерного времени после повторного включения электрического поля, эволюция как угла $\theta(x, z=0, \tau)$, так и безразмерной угловой скорости $\omega(x, z=0, \tau)$ в точности повторяют друг друга. Различия в поведении как угла $\theta$, так и угловой скорости $\omega$ начинаются с момента времени $\tau=6$. Так, в случае $B$ весь объем, занимаемый ЖК-фазой, вращается как единое целое, правда с различной угловой скоростью. Те домены, которые в случае $A$ вращались против часовой стрелки, в случае $B$ стали вращаться по часовой стрелке, правда с более высокой скоростью. К моменту времени $\tau_{R}($ in $)=10$ вращение ЖК-фазы, в обоих случаях $A$ и $B$, полностью прекратилось, при том, что в случае $B$ директор уже был равномерно сориентирован вдоль направления электрического поля E по всему объему, занимаемому ЖК-фазой.

Эволюция безразмерных сдвиговых компонент ТН $\sigma_{z x}(x, z=0, \tau)$ и $\sigma_{x z}(x, z=0, \tau)$ (см. рис. 9, $a$ и $\left.b\right)$ и нормальной компоненты $\sigma_{x x}(x, z=0, \tau)$ (см. рис. 10, $a$ ), к их равновесным значениям в трех точках, отстоящих на расстояниях $x=2.0$ (кривая 1), 3.0 (кривая 2) и 3.2 (кривая 3) от центра ЖК-канала, в течение первых 20 единиц безразмерного времени с момента повторного включения электрического поля

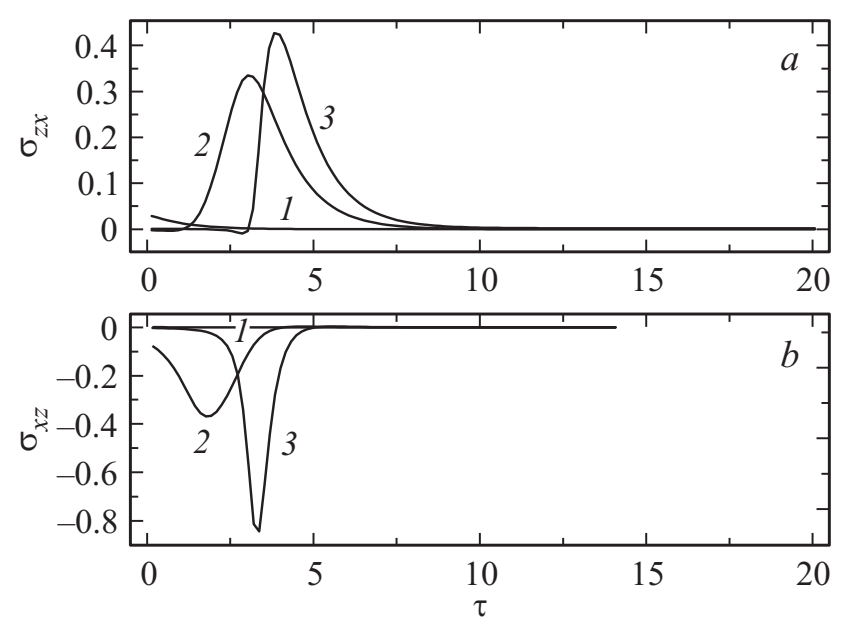

Рис. 9. Эволюция сдвиговых компонент ТН $\sigma_{z x}(x, z=0, \tau)(a)$ и $\sigma_{x z}(x, z=0, \tau)(b)$, в точках $(x=2.0)$ (кривые 1$),(x=3.0)$ (кривые 2) и $(x=3.2)$ (кривые 3 ) соответственно, к их равновесным распределениям $\sigma_{z x}^{e q}$ и $\sigma_{x z}^{e q}$, под действием сильного электрического поля $\mathbf{E}=-E \hat{\mathbf{k}} \quad(E \sim 1.05 \mathrm{~V} / \mu \mathrm{m}), \quad$ направленного под углом $\alpha=-1.57\left(\sim-89.96^{\circ}\right)$ к горизонтальным ограничивающим поверхностям ЖК-канала.
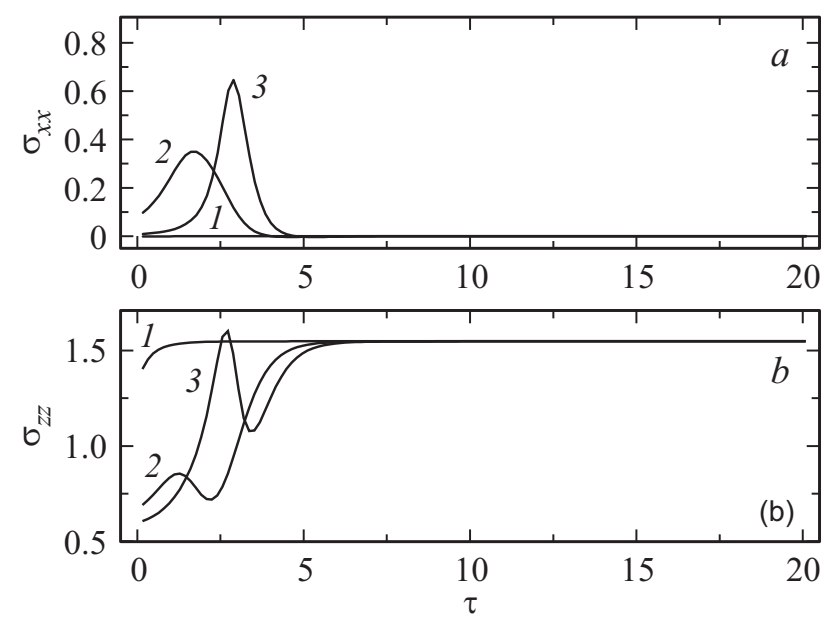

Рис. 10. То же, что на рис. 9, только для нормальных компонент ТН $\sigma_{x x}(x, z=0, \tau)(a)$ и $\sigma_{z z}(x, z=0, \tau)(b)$. 
$\mathbf{E}=-E \hat{\mathbf{k}} \quad(E \sim 1.05 \mathrm{~V} / \mu \mathrm{m}) \quad($ случай $B)$ показаны на рис. 9 и 10. Расчеты свидетельствуют о том, что абсолютные величины двух сдвиговых компонент ТН $\left|\sigma_{x z}\right|$ и $\left|\sigma_{z x}\right|$, а также нормальной компоненты $\left|\sigma_{x x}\right|$, на начальном этапе эволюции, соответствующем временам $\tau \sim 3 \div 4(\sim 18 \div 24 \mathrm{~ms})$, достигают максимальных значений $\sim 0.3 \div 0.8(\sim 6.6 \div 17.6 \mathrm{~Pa})$, а затем быстро убывают к нулю. В свою очередь, безразмерная нормальная компонента ТН $\sigma_{z z}(x, z=0, \tau)$ (рис. $\left.10, b\right)$ на начальном этапе эволюции, соответствующем временам $\tau \sim 3 \div 4(\sim 18 \div 24 \mathrm{~ms})$, осциллирует около значения $\sim 1.0(\sim 20 \mathrm{~Pa})$, а затем монотонно возрастает и на конечном этапе эволюции давление растяжения $\sigma_{z z}$, обусловленное сильным электрическим полем $\mathbf{E}=-E \hat{\mathbf{k}}$ $(E \sim 1.05 \mathrm{~V} / \mu \mathrm{m})$, достигает величины $\sim 1.5(\sim 33 \mathrm{~Pa})$, сравнимым с величиной компоненты ТН $\sigma_{z z}$ для случая поля $\mathbf{E}=E \hat{\mathbf{k}}$, направленного в противоположном направлении.

\section{4. Заключение}

В предлагаемой работе представлено численное исследование переориентации как поля директора $\hat{\mathbf{n}}$ и поля скорости $\mathbf{v}$, так и компонент тензора напряжения $\sigma_{i j}(i j=x, y, z)$ нематического жидкого кристалла (ЖК), инкапсулированного в прямоугольный канал, под действием сильного электрического поля $\mathbf{E}=E \hat{\mathbf{k}}$, направленного под углом относительно нормали $\hat{\mathbf{k}}$, направленной к планарно ориентированному ЖК-каналу. Численные расчеты, выполненные в рамках нелинейного обобщения классической теории ЭриксенаЛесли, показали, что при определенных соотношениях моментов и импульсов, действующих на единицу объема ЖК-фазы, и в случае $E \sim 1.05 \mathrm{~V} / \mu \mathrm{m}$ в процессе переориентации $\hat{\mathbf{n}}$ могут возникнуть переходные периодические структуры, если соответствующая мода искажения обладает наибыстрейшим откликом и, таким образом, подавляет все остальные моды, в том числе и однородные. Возникающие при этом вращающиеся домены способствовали уменьшению скорости диссипации энергии и, тем самым, создавали более выгодные, по сравнению с однородным поворотом, режимы переориентации поля директора. При этом были исследованы три динамических режима переориентации поля директора в нематике, образованном молекулами 4-n-пентил-4'-цианобифенила (5ЦБ), под действием сильного электрического поля $\mathbf{E}=E \hat{\mathbf{k}}$. Первый режим соответствовал включению $\mathbf{E}$, направленному в положительном направлении, перпендикулярно однородно ориентированному нематику, второй соответствовал режиму с выключенным полем, $\mathbf{E}=0$, и наконец, третий режим соответствовал повторному включению $\mathbf{E}$, но направленному в отрицательном направлении относительно нормали к планарно ориентированному ЖК-каналу.
Мы полагаем, что данная работа проливает свет на неизученные аспекты динамики переориентации поля директора в микроскопических ЖК-каналах под действием сильного электрического поля.

\section{Приложение. Моменты и компоненты тензора напряжений}

Мы рассмотрим нематический ЖК, где поле директора задано вектором $\hat{\mathbf{n}}=\left(n_{x}, 0, n_{z}\right)=(\cos \theta, 0, \sin \theta)$, а баланс вращательных моментов, действующих на единицу объема ЖК-фазы, состоит из $[7,10] \mathbf{T}_{\text {elast }}=T_{\text {elast }} \hat{\mathbf{j}}=$ $=\frac{\delta \mathscr{W}_{\mathrm{F}}}{\delta \hat{\mathbf{n}}} \times \hat{\mathbf{n}}-$ упругого, $\mathbf{T}_{\text {vis }}=T_{\text {vis }} \hat{\mathbf{j}}=\frac{\delta \mathscr{R}^{\text {vis }}}{\delta \tilde{\mathbf{n}}_{t}} \times \hat{\mathbf{n}}-$ вязкого и $\mathbf{T}_{\mathrm{el}}=T_{\mathrm{e}} \hat{\mathbf{j}}=\frac{\delta \psi_{\mathrm{el}}}{\delta \hat{\mathbf{n}}} \times \hat{\mathbf{n}}-$ электрического вкладов. Здесь $\mathscr{W}_{\mathrm{F}}=\frac{1}{2}\left[K_{1}(\nabla \cdot \hat{\mathbf{n}})^{2}+K_{3}(\hat{\mathbf{n}} \times \nabla \times \hat{\mathbf{n}})^{2}\right]-$ плотность упругой энергии, приходящейся на единицу объема ЖК-фазы, $\psi_{e l}=-\frac{1}{2} \epsilon_{0} \epsilon_{a}(\hat{\mathbf{n}} \cdot \mathbf{E})^{2}-$ плотность электрической энергии,

$$
\begin{aligned}
\mathscr{R}^{\mathrm{vis}}= & \alpha_{1}\left(\hat{\mathbf{n}} \cdot \mathbf{D}_{s} \cdot \hat{\mathbf{n}}\right)^{2}+\gamma_{1}\left(\hat{\mathbf{n}}_{\mathrm{t}}-\mathbf{D}_{a} \cdot \hat{\mathbf{n}}\right)^{2} \\
& +2 \gamma_{2}\left(\hat{\mathbf{n}}_{\mathrm{t}}-\mathbf{D}_{a} \cdot \hat{\mathbf{n}}\right) \cdot\left(\mathbf{D}_{s} \cdot \hat{\mathbf{n}}-\left(\hat{\mathbf{n}} \cdot \mathbf{D}_{s} \cdot \hat{\mathbf{n}}\right) \hat{\mathbf{n}}\right) \\
& +\alpha_{4} \mathbf{D}_{s}: \mathbf{D}_{s}+\left(\alpha_{5}+\alpha_{6}\right)\left(\hat{\mathbf{n}} \cdot \mathbf{D}_{s} \cdot \mathbf{D}_{s} \cdot \hat{\mathbf{n}}\right)
\end{aligned}
$$

- вязкий вклад в полную функцию Рэлея $\mathscr{R}=\mathscr{R}^{\mathrm{vis}}$. Здесь $K_{1}$ и $K_{3}-$ коэффициенты упругости Франка, соответствующие поперечному и продольному изгибам, $\mathbf{D}_{s}=\frac{1}{2}\left[\nabla \mathbf{v}+(\nabla \mathbf{v})^{\mathrm{T}}\right]$ и $\mathbf{D}_{a}=\frac{1}{2}\left[\nabla \mathbf{v}-(\nabla \mathbf{v})^{\mathrm{T}}\right]-$ симметричный и антисимметричный вклады в тензор $\nabla \mathbf{v}, \quad \hat{\mathbf{n}}_{t}=\frac{d \hat{\mathbf{n}}}{d t}-$ материальная производная, $\alpha_{i}(i=1, \ldots, 6)$ - коэффициенты вязкости Лесли, а $\gamma_{1}=\alpha_{3}-\alpha_{2}, \gamma_{2}=\alpha_{3}+\alpha_{2}-$ коэффициенты вращательной вязкости.

Безразмерный тензор напряжений (ТH) представляет собой сумму, состоящую из упругих $\left(\sigma^{\text {elast }}\right)$, вязких $\left(\sigma^{\mathrm{vis}}\right)$ и электрических $\left(\sigma^{\mathrm{el}}\right)$ вкладов за вычетом $P \mathscr{L}$. Компоненты упругого ТН имеют вид

$$
\begin{aligned}
& \sigma_{x x}^{\text {elast }}=\delta_{1}\left(-\Delta_{1} \theta_{, x}^{2}+\Delta_{3} \theta_{, x} \theta_{, z}\right), \\
& \sigma_{z z}^{\text {elast }}=\delta_{1}\left(-\Delta_{2} \theta_{, z}^{2}+\Delta_{3} \theta_{, x} \theta_{, z}\right), \\
& \sigma_{x z}^{\text {elast }}=\delta_{1}\left(-\Delta_{1} \theta_{, x} \theta_{, z}+\Delta_{3} \theta_{, z}^{2}\right), \\
& \sigma_{z x}^{\text {elast }}=\delta_{1}\left(\Delta_{2} \theta_{, x} \theta_{, z}+\Delta_{3} \theta_{, x}^{2}\right),
\end{aligned}
$$

где

$$
\begin{gathered}
\Delta_{1}=\sin ^{2} \theta+K_{31} \cos ^{2} \theta, \quad \Delta_{2}=\cos ^{2} \theta+K_{31} \sin ^{2} \theta, \\
\Delta_{3}=\frac{1-K_{31}}{2} \sin 2 \theta, \quad K_{31}=K_{3} / K_{1} .
\end{gathered}
$$

Безразмерные компоненты вязкого ТН имеют вид:

$$
\sigma_{i j}^{\mathrm{vis}}=f_{i j}^{1, \text { vis }} \psi_{, x x}+f_{i j}^{2, \mathrm{vis}} \psi_{, z z}+f_{i j}^{3, \mathrm{vis}} \psi_{, x z}+f_{i j}^{4, \mathrm{vis}} \psi_{, z x},
$$


где

$$
\begin{gathered}
f_{x x}^{1, \text { vis }}=-\frac{\sin 2 \theta}{4}\left(\frac{2 \alpha_{1}}{\gamma_{1}}+\gamma^{2} \cos 2 \theta\right), \\
f_{x x}^{2, \text { vis }}=-f_{x x}^{1, \text { vis }},
\end{gathered}
$$

$$
f_{x x}^{3, \text { vis }}=\frac{1}{\gamma_{1}}\left[\alpha_{1} \cos 2 \theta \cos ^{2} \theta+\left(\alpha_{5}+\alpha_{6}\right) \cos ^{2} \theta+\alpha_{4}\right]
$$$$
-\frac{\gamma^{2}}{4} \sin ^{2} \theta
$$$$
f_{x x}^{4, \text { vis }}=-\frac{\gamma}{2}\left[\sin 2 \theta\left(\Delta_{1} \theta_{, x x}+\Delta_{2} \theta_{, z z}\right)\right.
$$$$
\left.+\Delta_{5}\left(\theta_{, x}^{2}+2 \theta_{, x z}-\theta_{, z}^{2}-2 \theta_{, x} \theta_{, z}\right)\right],
$$$$
f_{z z}^{1, \text { vis }}=-\frac{\sin 2 \theta}{4}\left(2 \frac{\alpha_{1}}{\gamma_{1}} \cos ^{2} \theta-\gamma^{2} \cos 2 \theta\right) \text {, }
$$$$
f_{z z}^{2 \text {,vis }}=-f_{z z}^{1, \text { vis }} \text {, }
$$

$$
f_{z z}^{3, \text { vis }}=\frac{1}{\gamma_{1}}\left[\alpha_{1} \cos 2 \theta \sin ^{2} \theta-\left(\alpha_{5}+\alpha_{6}\right) \cos ^{2} \theta-\alpha_{4}\right]
$$$$
+\frac{\gamma^{2}}{4} \sin ^{2} \theta, \quad f_{z z}^{4, \text { vis }}=-f_{x x}^{4, \text { vis }},
$$$$
f_{x z}^{1, \text { vis }}=\frac{1}{4 \gamma_{1}}\left(-\alpha_{1} \sin ^{2} 2 \theta+2 \gamma_{2} \cos 2 \theta-2 \alpha_{4}\right.
$$

$$
\left.-\alpha_{5}-\alpha_{6}-\gamma_{1}\right)+\Delta_{6}^{2}
$$

$$
f_{x z}^{2, \text { vis }}=\frac{1}{4 \gamma_{1}}\left(\alpha_{1} \sin ^{2} 2 \theta+2 \alpha_{4}+\alpha_{5}+\alpha_{6}-\gamma_{1}\right)+\Delta_{7},
$$$$
f_{x z}^{3, \mathrm{vis}}=-\frac{\sin 4 \theta}{4}\left(\frac{\alpha_{1}}{\gamma_{1}}+\gamma^{2}\right),
$$$$
f_{x z}^{4, \text { vis }}=-\Delta_{6}\left(\Delta_{1} \theta_{, x x}+\Delta_{2} \theta_{, z z}\right)
$$$$
-\Delta_{3} \Delta_{6}\left(\theta_{, x}^{2}+2 \theta_{, x z}-\theta_{, z}^{2}-2 \theta_{, x} \theta_{, z}\right),
$$

$$
f_{z x}^{1, \text { vis }}=-\frac{1}{4 \gamma_{1}}\left(\alpha_{1} \sin ^{2} 2 \theta+2 \alpha_{4}+\alpha_{5}+\alpha_{6}-\gamma_{1}\right)+\Delta_{6}^{2},
$$$$
f_{z x}^{2, \text { vis }}=\frac{1}{4 \gamma_{1}}\left(\alpha_{1} \sin ^{2} 2 \theta+2 \gamma_{2} \cos 2 \theta+2 \alpha_{4}\right.
$$$$
\left.+\alpha_{5}+\alpha_{6}+\gamma_{1}\right)-\Delta_{8}^{2}, \quad f_{z x}^{3, \text { vis }}=-f_{x z}^{3, \text { vis }},
$$$$
f_{z x}^{4, \text { vis }}=\Delta_{8}\left(\Delta_{1} \theta_{, x x}+\Delta_{2} \theta_{, z z}\right)
$$

$$
+\Delta_{8} \Delta_{3}\left(\theta_{, x}^{2}+2 \theta_{, x z}-\theta_{, z}^{2}-2 \theta_{, x} \theta_{, z}\right)
$$

где

$$
\begin{gathered}
\Delta_{5}=\frac{1-K_{31}}{2} \sin ^{2} 2 \theta, \quad \Delta_{6}=\frac{1}{2}(1-\gamma \cos 2 \theta), \\
\Delta_{7}=\frac{1}{4}\left(1-\gamma^{2} \cos ^{2} 2 \theta\right), \quad \Delta_{8}=\frac{1}{2}(1+\gamma \cos 2 \theta) .
\end{gathered}
$$

Безразмерный аналог уравнения Навье-Стокса $\rho \frac{d \mathbf{v}}{d t}=\nabla \cdot \sigma$ принимает вид

$$
\delta_{2}\left[(\Delta \psi)_{, \tau}+\psi_{, z}(\Delta \psi)_{, x}-\psi_{, x}(\Delta \psi)_{, z}\right]=\hat{\mathscr{L}} \psi+\mathscr{F},
$$

где $\Delta \psi=\psi_{, x x}+\psi_{, z z}, \mathscr{F}=\mathscr{F}_{\text {elast }}+\mathscr{F}_{\text {el }}, \mathrm{a}$

$$
\begin{gathered}
\mathscr{F}_{\text {elast }}=\left(\sigma_{x x}^{\text {elast }}+\sigma_{z z}^{\text {elast }}\right)_{, x z}+\left(\sigma_{z x}^{\text {elast }}\right)_{, z z}-\left(\sigma_{x z}^{\text {elast }}\right)_{, x x}, \\
\mathscr{F}_{\text {el }}=-\left(\sigma_{z z}^{\text {el }}\right)_{, x z},
\end{gathered}
$$

и

$$
\sigma_{z z}^{\mathrm{el}}=\bar{E}^{2} \sin \alpha\left(\frac{\epsilon_{\perp}}{\epsilon_{a}}+\sin ^{2} \theta\right) .
$$

Оператор

$$
\begin{aligned}
\hat{\mathscr{L}} \psi= & a_{1} \psi_{, z z z z}+a_{2} \psi_{, x z z z}+a_{3} \psi_{, x x z z}+a_{4} \psi_{, x x x z} \\
& +a_{5} \psi_{, x x x x}+a_{6} \psi_{, z z z}+a_{7} \psi_{, x z z}+a_{8} \psi_{, x x z} \\
& +a_{9} \psi_{, x x x}+a_{10} \psi_{, z z}+a_{11} \psi_{, x z}+a_{12} \psi_{, x x},
\end{aligned}
$$

где

$$
\begin{gathered}
a_{1}=f_{z x}^{2, \text { vis }}, \quad a_{2}=f_{z x}^{3, \text { vis }}+f_{x x}^{2, \text { vis }}-f_{z z}^{2, \text { vis }}, \\
a_{3}=f_{z x}^{1, \text { vis }}-f_{x z}^{2, \text { vis }}+f_{x x}^{3, \text { vis }}-f_{z z}^{3 \text {,vis }}, \\
a_{4}=f_{x x}^{1, \text { vis }}-f_{z z}^{1, \text { vis }}-f_{x z}^{3, \text { vis }}, \\
a_{5}=-f_{x z}^{1, \text { vis }}, \quad a_{6}=f_{x x, z}^{2, \text { vis }}-f_{z z}^{2, \text { vis }}+2 f_{z x, z}^{2, \text { vis }}, \\
a_{7}=f_{x x, z}^{2, \text { vis }}-f_{z z, z}^{2, \text { vis }}+f_{x x, x}^{3, \text { vis }}-f_{z z, z}^{3, \text { vis }}+2 f_{z x, z}^{3, \text { vis }}-2 f_{x z, x}^{3, \text { vis }}, \\
a_{8}=f_{x x, x}^{1, \text { vis }}-f_{z z, x}^{1, \text { vis }}+f_{x x, z}^{3, \text { vis }}-f_{z z, z}^{3, \text { vis }}+2 f_{z x, z}^{1, \text { vis }}-2 f_{x z, x}^{3, \text { vis }}, \\
a_{9}=f_{x x, z}^{1, \text { vis }}-f_{z z, z}^{1, \text { vis }}-2 f_{x z, x}^{1, \text { vis }}, \\
a_{10}=f_{x x, x z}^{2, \text { vis }}-f_{z z, x z}^{2, \text { vis }}+f_{z x, z z}^{2, \text { vis }}-f_{x z, x x}^{2, \text { vis }}, \\
a_{11}=f_{x x, x z}^{3, \text { vis }}-f_{z z, x z}^{3, \text { vis }}+f_{z x, z z}^{3, \text { vis }}-f_{x z, x x}^{3, \text { vis }}, \\
a_{12}=f_{x x, x z}^{1, \text { vis }}-f_{z z, x z}^{1, \text { vis }}+f_{z x, z z}^{1, \text { vis }}-f_{x z, x x}^{1, \text { vis } .}
\end{gathered}
$$

\section{Список литературы}

[1] S. Lee, R. An, J.A. Hunt. Nature Nanotech. 5, 412 (2010).

[2] S. Samitsu, Y. Takanishi, J. Yamamoto. Nature Mater. 9, 816 (2010).

[3] H. Ren, Su Xu, S-T. Wu. Lab. Chip. 13, 100 (2013).

[4] R. Daugla, S. Cagri Kayi, Ch.N. Baroud. Proc. Natl. Acad. Sci. 110, 853 (2013).

[5] P.G. de Gennes, J. Prost. The physics of liquid crystals. Oxford Univ. Press. Oxford (1995). 400 p.

[6] A. Sugimura, A.V. Zakharov. Phys. Rev. E 84, 021703 (2011).

[7] A.A. Vakulenko, A.V. Zakharov. Phys. Rev. E 88, 022505 (2013).

[8] J.L. Ericksen. Arch. Ration. Mech. Anal. 4, 231 (1960).

[9] F.M. Leslie. Arch. Ration. Mech. Anal. 28, 265 (1968).

[10] А.В. Захаров, А.А. Вакуленко, С.В. Пасечник. ФТТ 58, 1851 (2016).

[11] И.С. Березин, Н.Р. Жидков. Методы вычислений. Физматгиз, М. (1964). 464 c. 